\title{
Propagating Activation during Oscillations and Evoked Responses in Neocortical Slices
}

\author{
Jian-young Wu, Li Guan, and Yang Tsau \\ Institute for Cognitive and Computational Sciences, Georgetown University Medical Center, Washington, DC 20007
}

Population activity in the cortex is poorly understood. In this report we use voltage-sensitive dye imaging to examine the spatiotemporal patterns of a $7-10 \mathrm{~Hz}$ oscillation in neocortical slices from rat somatosensory areas. This oscillation appeared as a component of spontaneous epochs when the preparation was bathed in low [Mg] artificial CSF (ACSF) (Silva et al., 1991). Each epoch started with a synchronized spike, and 3-200 cycles of oscillation emerged afterward. Voltage-sensitive dye imaging revealed that the oscillations in the local field potential recordings were actually caused by a propagating population activation. This activation propagated in a relatively uniform size (not expanding). We call this confined, propagating activation a "dynamic ensemble." During each oscillation cycle, one (occasionally two) dynamic ensemble(s) appeared in the slice and was sustained for 60-200 msec. Dynamic ensembles propagated at $\sim 30 \mathrm{~mm} / \mathrm{sec}$; the activity could propagate in both directions in cortical slices. The propagation consisted in part of "jumps," the locations of which were not fixed. Dynamic ensembles were distinguishable from the epileptiform spikes that occurred in low [Mg] ACSF. Population events similar to dynamic ensembles were also evoked under conditions of unaltered excitability (slice in normal ACSF) by electrical stimulation that activated a low density of neurons in a large area. Our data suggest that self-sustained, spatially confined, and propagating dynamic ensembles might be related to the epoch oscillations in somatosensory cortex seen in vivo (Nicolelis et al., 1995) and thus resemble one form of population activation in the neocortex.

Key words: oscillation; somatosensory cortex; dynamic ensemble; optical recordings; voltage-sensitive dyes; population activity; synchronous events; cortical slices
Oscillations are common population events in the CNS (Gray, 1994). Synchrony during oscillations on different spatial scales has been extensively observed (Engel et al., 1991; Llinas and Ribary, 1993; Steriade et al., 1993; Nicolelis et al., 1995; Murthy and Fetz, 1996). It has been reported that over short distances of $1 \mathrm{~mm}$ or less, neighboring neurons dynamically form synchronized assemblies (Gerstein and Aertsen, 1985; Gerstein et al., 1989). Such dynamically organized neuronal assemblies have been a subject of interest (Singer, 1985; Palm, 1990; Churchland and Sejnowski, 1992; Amit, 1995; Freeman, 1995; Crick, 1996; Nicolelis et al., 1997). Imaging the spatiotemporal patterns of population oscillations may help to understand the correlation between long distance synchrony and localized neuronal assemblies.

Optically imaging an oscillation in the in vivo cortex is technically difficult. Intrinsic optical signals (Ts'o et al., 1990; Malonek and Grinvald, 1996; Wang et al., 1996) are too slow for imaging most oscillations. Voltage-sensitive dye imaging is fast, but the signals (without averaging) are usually smaller than the artifacts of brain pulsation. In measurements from rodent somatosensory cortex, the signal-to-noise ratio only allowed a resolution of epileptiform events in single trials. The evoked response to whis-

\footnotetext{
Received Oct. 1, 1998; revised March 25, 1999; accepted March 25, 1999.

This work was supported by National Institutes of Health Grants NS31425 and DAMD17-93-V3018 and by a grant from the Epilepsy Foundation of America. We thank Drs. L. B. Cohen, B. Connors, G. B. Ermentrout, D. Golomb, J. R. Metherate, P. Rauschecker, and B. Tian for critically reading this manuscript and/or helpful discussions; Dr. W. Chen for helping with experiments; Dr. T. Kiemel for assisting with the numerical analysis; and Ms. Schaefer for technical assistance with both the experiments and the preparation of this manuscript.

Correspondence should be addressed to Dr. Jian-young Wu, Georgetown University, New Research Building, WP-26, 3970 Reservoir Road, Northwest, Washington, DC 20007.

Copyright (C) 1999 Society for Neuroscience $\quad 0270-6474 / 99 / 195005-11 \$ 05.00 / 0$
}

ker movement was $\sim 1 / 10$ th the size of the signals of epileptiform events (London et al., 1989). Even with improvements in the recording techniques, averaging was required to resolve a sensory-evoked response in the cortex (Kleinfeld and Delaney, 1996; however, see Arieli et al., 1996, on cat visual cortex). Furthermore, voltage-sensitive dye recordings from turtle visual cortex (Prechtl et al., 1997) showed that the oscillation signals were approximately five times smaller than were the sensoryevoked response. Thus, examining the oscillation patterns from mammalian cortex in vivo would require substantial technical improvement [e.g., new dyes (Glaser et al., 1998)].

Imaging in brain slices has a large signal-to-noise ratio, allowing the resolution of oscillation patterns in single trials. Voltagesensitive dye imaging has been used in studying population activation in brain slices (e.g., Grinvald et al., 1982; Albowitz and Kuhnt, 1991; Colom and Saggau, 1994; Nelson and Katz, 1995; Demir et al., 1998; Tsau et al., 1998); however, the spatiotemporal pattern of oscillations in adult neocortical slices has not been reported, probably because in adult slices oscillations have not been clearly distinguished from epileptiform activity.

A cortical slice preserves a complex local network with a large number of intrinsic bursting neurons (Connors and Gutnick, 1990) that may serve as pacemakers for the oscillations (Silva et al., 1991). The 7-10 Hz oscillation in slices from rat somatosensory cortex (Silva et al., 1991) is similar to the oscillations occurring in vivo (Nicolelis et al., 1995). In this report we use this preparation to address two questions. (1) With many intrinsic bursters, does this oscillation start in one spot (a local pacemaker) and propagate to other areas or start from many spots, distributed in an interactive pattern? (2) Do active neurons form localized clusters during this oscillation? 


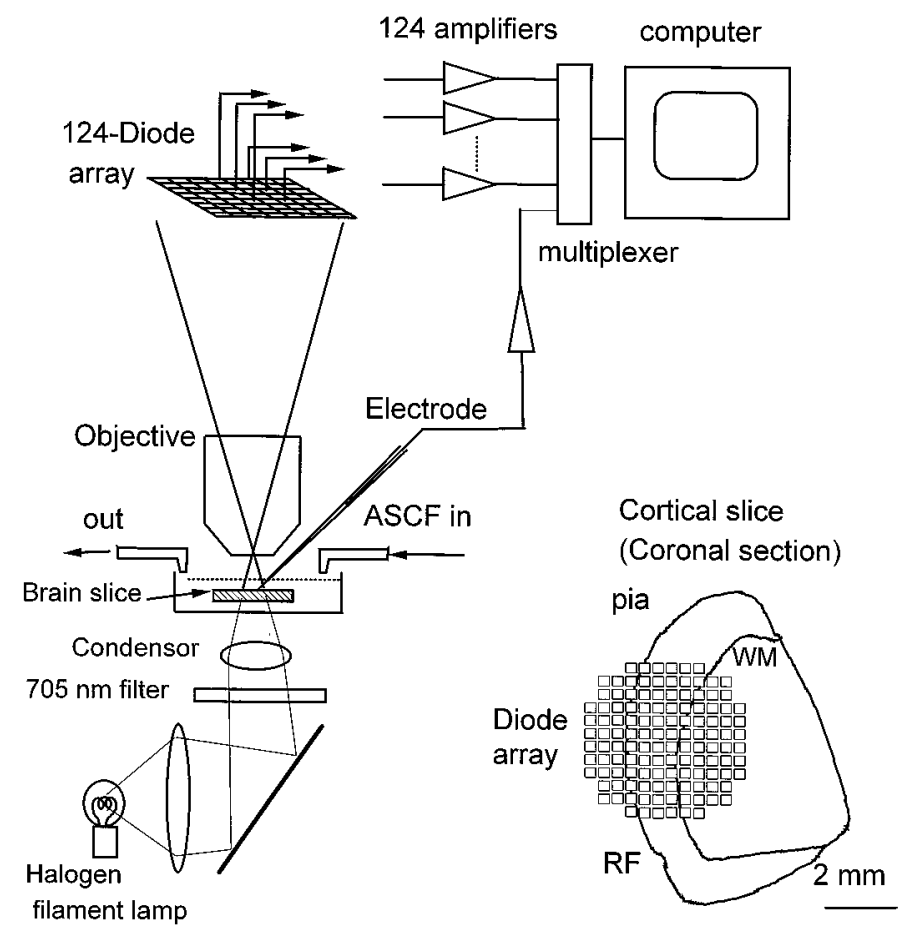

Figure 1. Apparatus for optical imaging. The slice preparation was continuously perfused with low $[\mathrm{Mg}] \mathrm{ACSF}$ in a submerge chamber on the stage of a modified Leitz Orthoplan microscope (Wetzlar, Germany). The chamber and the perfusing solution were heated at $26^{\circ} \mathrm{C}$. To reduce the vibration noise for optical recordings, we mounted the microscope on an air table and paused the perfusion during optical recording trials. We made absorption measurements using transmission illumination. Light from a halogen tungsten filament bulb $(12 \mathrm{~V} ; 100 \mathrm{~W})$ was collected and illuminated the preparation through a $705 \pm 60 \mathrm{~nm}$ filter. The image was formed by a $2.8 \times(\mathrm{NA}, 0.05)$ objective. With this optical magnification, the six laminae of the cortex were projected to five rows of photodetectors (lower right diagram), and a 4-mm-wide cortical section was monitored by $\sim 50$ neighboring detectors. $R F$, Rhinal fissure; $W M$, white matter.

\section{MATERIALS AND METHODS}

Cortical slices were harvested from postnatal day 21 to adult Sprague Dawley rats of both sexes. Following the National Institutes of Health guidelines, the animals were deeply anesthetized with halothane and quickly decapitated using a Stoelting small animal decapitator. The whole brain was moved into cold $\left(0-4^{\circ} \mathrm{C}\right)$ artificial CSF (ACSF) containing (in mM): $\mathrm{NaCl}, 132 ; \mathrm{KCl}, 3 ; \mathrm{CaCl}_{2}, 2 ; \mathrm{MgSO}_{4}, 2 ; \mathrm{NaH}_{2} \mathrm{PO}_{4}, 1.25$; $\mathrm{NaHCO} 3,26$; and dextrose 10 , saturated with $95 \% \mathrm{O} 2 / 5 \% \mathrm{CO}_{2}, \mathrm{pH}=$ 7.4. The brain was chilled in the cold ACSF for $90 \mathrm{sec}$ before blocking and slicing.

The Paxinos and Watson (1986) stereotaxic atlas for rat brain was used to locate the cortical areas. The slicing angles were determined by the angles with which the brain was blocked. The tissue block was glued on a vibratome stage (752M Vibroslice; Campden Instruments, Sarasota, FL). Slices of $400 \mu \mathrm{m}$ thickness from somatosensory areas were obtained. The slices were then transferred into a holding chamber, immersed in ACSF, and oxygenated with $95 \% \mathrm{O} 2 / 5 \% \mathrm{CO}_{2}$ at room temperature for at least $2 \mathrm{hr}$. The slices were then perfused in ACSF containing the oxonol voltage-sensitive dye RH 479 [first synthesized by R. Hildesheim and A. Grinvald and kindly provided by Dr. L. Loew (University of Connecticut, Farmington, CT) as JPW 1131] at $0.02 \mathrm{mg} / \mathrm{ml}$ for $1 \mathrm{hr}$. The stained preparation was then perfused with dye-free ACSF at $29^{\circ} \mathrm{C}$ for 30 min before the measurements. Low $[\mathrm{Mg}]$ ACSF was used in some experiments. This ACSF was made with the same components and concentrations as the ACSF described above, but the concentration of $\mathrm{MgSO}_{4}$ was reduced from 2 to $0.1 \mathrm{~mm}$.

Optical imaging was performed with a 124-element photodiode array (Centronics, Newbury Park, CA) at a frame rate of 1000 frames/sec (Fig. 1). A $2.8 \times$ [numerical aperture (NA), 0.05] objective was used to project the image of the preparation to the array. On the array each detector received light from a $0.3 \times 0.3 \mathrm{~mm}^{2}$ area of the slice. The photocurrent of dye-related absorption signals $(705 \mathrm{~nm})$ from each photodetector was individually amplified through a two-stage amplifier system. The first stage of amplification performed a current-to-voltage conversion using a feedback resistor of $1 \mathrm{M} \Omega$. The signals were then amplified and filtered by a second stage amplifier before digitizing. This second stage provides a voltage gain of 200 or 2000 , high-pass filtering with a $100 \mathrm{msec}$ time constant, and a four-pole Bessel analog low-pass filter with a $300 \mathrm{~Hz}$ corner frequency. [The amplifiers are commercially available as NeuroPlex systems (OptImaging, Fairfield, CT).] This parallel amplifier arrangement (Wu and Cohen, 1993) allows a low dark noise $\left(10^{-6}\right.$ of the illumination intensity), a large dynamic range (17-21 bits), and a fast sampling rate. The photocurrent on each diode was $\sim 100 \mathrm{nA}$. The optical signal size $(\mathrm{d} I / I)$ for the oscillations was $\sim 0.05-0.2 \%(5-20 \times$ $10^{-4}$ ). The signal-to-noise ratio at our light level was $\sim 5$ for oscillation signals, so that averaging was not necessary. Optical recording trials were 8-16 sec long; on each slice we record 4-10 trials (with a total exposure of $<150 \mathrm{sec}$ ). Phototoxicity was not a concern with this level of exposure time and intensity. [For additional details about the apparatus, see Wu et al. (1999).]

Glass micropipettes or tungsten lacquer-coated microelectrodes were used to sample local field potentials (LFPs) and the spiking of individual neurons simultaneously. Glass electrodes were made from borosilicate glass containing a capillary fiber $(0.75 \mathrm{~mm}$ inner diameter; $1.0 \mathrm{~mm}$ outer diameter), were filled with $1 \mathrm{M} \mathrm{NaCl}$, and had resistance ranging from 2 to $5 \mathrm{M} \Omega$. In most experiments, the recording electrodes were placed in layer II of the cortex. Tips of electrodes were inserted $\sim 100 \mu \mathrm{m}$ into the slice. LFP recordings were made with the use of two intracellular amplifiers (IE201; Warner Instrument Corporation, Hamden, CT). In some experiments, one electrode was used as a stimulus electrode when the amplifier probe was set to electrode input mode. Stimuli consisted of rectangular current pulses $10-30 \mathrm{~V}$ in amplitude and $50-200 \mu$ sec in duration. Electrical recordings were digitized and stored concurrently with the optical images. For long-term electrical records, data were also stored on videotapes through a digital recorder (CRC VR-100) and then transferred to a personal computer (PC) for further analysis.

Signals from the photodiode array and electrodes were multiplexed and digitized with a DAP3200e/214 12-bit data acquisition board installed in a pentium PC and two multiplexer boards (Microstar Laboratories, Bellevue, WA) controlled by a program written in BASIC. Data were acquired and directly transferred into a hard drive.

The optical data were analyzed using the program NeuroPlex (A. Cohen and C. Falk; OptImaging). NeuroPlex displays the data in the form of traces for numerical analysis or pseudocolor images for visualizing the spatiotemporal pattern. The digitized data were further filtered by NeuroPlex, low-pass, $300 \mathrm{~Hz}$ (Butterworth) and high-pass, $1.2 \mathrm{~Hz}$ (RC filter) before displaying. The pseudocolor display was made using variable scaling and contour display. In the variable-scaling mode, the data from each diode are first normalized to their own maximum, and then each normalized data point is assigned a color according to a linear color scale $(0=$ deep blue and $1=$ red $)$. This kind of scaling best preserves the timing information in the signals. The contour mode spatially low-pass filters the raw data, so that the areas of equal signal amplitude are assigned the same color, and the amplitude difference between neighboring diodes is interpolated; the pseudocolor maps are not displayed in discrete pixels.

Numerical analysis, fast Fourier transformation (FFT), and auto- and cross-correlation were performed using MatLab (Mathworks, Natick, MA).

\section{RESULTS}

In this report we examine dynamically organized, multineuronal ensemble activity during oscillations. We are interested in whether this kind of activity exists during a spontaneous oscillation and can also be evoked as an all-or-none event. Here we first examine spatiotemporal patterns of $7-10 \mathrm{~Hz}$ oscillations in rat somatosensory cortical slices and then explore whether similar activation can be evoked by specific methods of stimulation.

\section{Optical signals of population oscillations}

The oscillations appear in cortical slices from the somatosensory areas as part of the spontaneous epochs that occur when the preparation is bathed in low [Mg] ACSF (Silva et al., 1991; Flint and Connors, 1996) (Fig. 2A). Each epoch started with a synchro- 


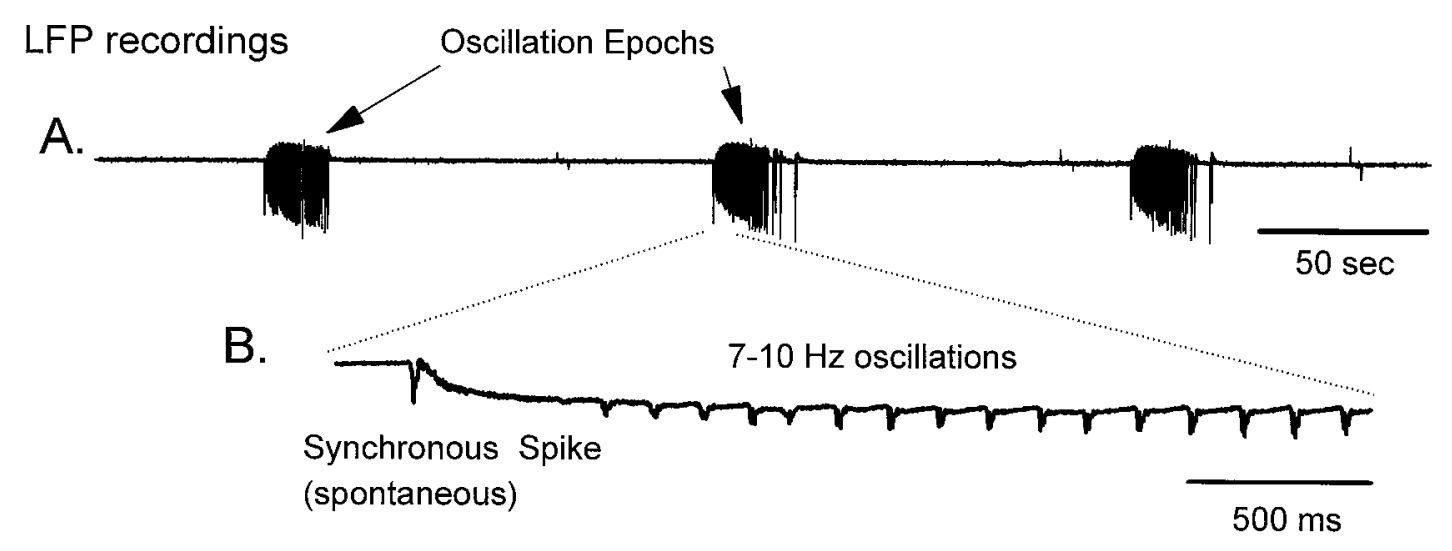

Figure 2. Epoch oscillations measured with an LFP electrode. A, When a cortical slice is perfused in low [Mg] ACSF, epochs of oscillations spontaneously occur. This recording is made by an electrode placed in cortical layer II, after $2 \mathrm{hr}$ of perf usion in low [Mg] ACSF. $B$, An expanded display shows that an epoch started with a spontaneous synchronous spike and $7-10 \mathrm{~Hz}$ oscillations gradually emerged a few seconds later. In different preparations the epochs consisted of 3-200 cycles of oscillation. In all preparations tested $(n>50)$, all oscillations appeared in spontaneous epochs, and each epoch started with a synchronous spike. However, in seven preparations, there were only one or two oscillation cycles in each epoch. The number of oscillation cycles in each epoch appeared to increase the longer the interepoch intervals were.

\section{A. LFP and optical recordings}

\section{1,2: Optical signal}

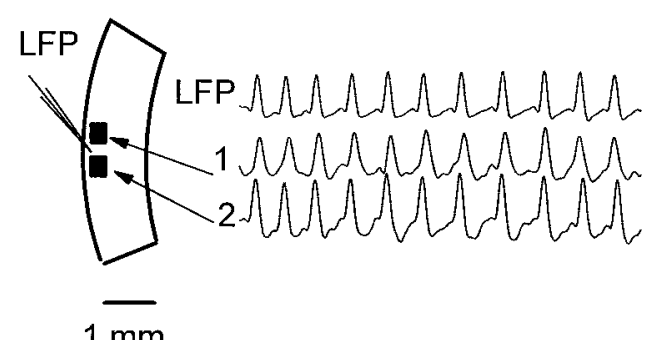

\section{B. Cross correlation}

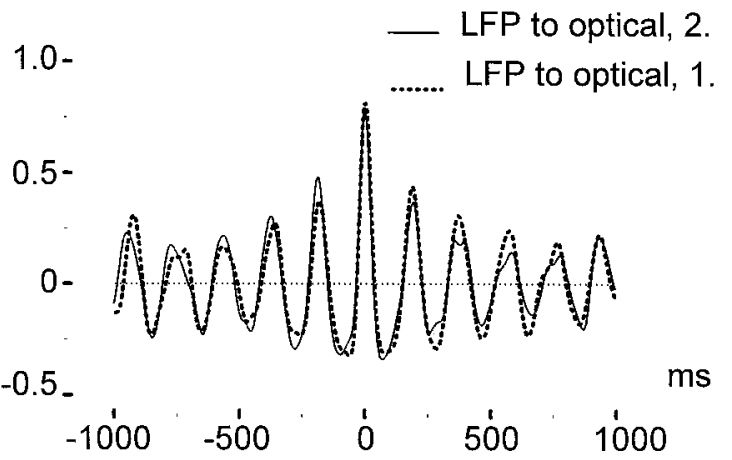

Figure 3. Comparison of electrical (LFP) and voltage-sensitive dye signals. $A$, During oscillations, the LFP signals and optical signals from neighboring sites were highly correlated. The recording sites were $\sim 0.5 \mathrm{~mm}$ apart, and there was a small phase difference between the different locations. $B$, The cross-correlation between LFP signals and the two optical detectors was high during the oscillations.

nous spike, and oscillations emerged afterward (Fig. $2 B$ ). In each epoch there was a variable number (usually 3-200) of oscillation cycles. The low-amplitude peaks of oscillations appeared to emerge from low-amplitude, asynchronized activities. As the epoch progressed, the oscillation frequency gradually decreased while the amplitude increased (Fig. $2 B$ ). At $26^{\circ} \mathrm{C}$, the frequency was $5-8 \mathrm{~Hz}$.

We found that these LFP signals were highly correlated with the voltage-sensitive dye signals. When LFP and voltage-sensitive dye signals were simultaneously recorded, the waveform of LFP and optical detectors at nearby areas was similar and highly correlated (Fig. 3). The optical signals were wavelengthdependent, with a maximum signal $(\mathrm{d} I / I)$ at $705 \mathrm{~nm}$ and no signal at $670 \mathrm{~nm}$. These are major features of the signal from RH 479, the oxonol voltage-sensitive dye. The amplitude of an optical signal is proportional to the depolarization of all the stained membranes under each detector (for review, see $\mathrm{Wu}$ and Cohen, 1993). These signals had a rapid time course and thus could not have come from other optical sources, such as activity-related intrinsic optical signals (Hochman et al., 1995; Malonek and Grinvald, 1996; Yuste et al., 1997; Peterson et al., 1998). These results indicate that the optical signals faithfully represent the population neuronal activity, which allows us to examine this oscillation from many neighboring locations optically.

\section{Spatial distribution of the oscillations}

In the measurement shown in Figure 4, a 4-mm-wide cortical slice was imaged (Fig. $4 A$ ), in which $\sim 50$ neighboring locations were recorded simultaneously (Fig. $4 A, B$ ). In these measurements we began an optical recording trial when an epoch occurred. In most of the preparations examined $(n>20)$, all locations oscillated at the same overall frequency (Fig. $4 C$ ). To our surprise, the correlations between two locations did not decrease significantly with distance, although there were large phase shifts at a large distance (Fig. 5A). The correlation was better between two points within one vertical "beam" than between two points separated by the same distance but in a tangential direction (Fig. 5B), suggesting that cortical columns might serve as synchronized units during oscillations.

Autocorrelations of the signals from five locations (Fig. 5B) suggested that the entire preparation was dominated by one frequency. However, the autocorrelation peaks were no longer superimposed after two cycles in either direction from 0 time, indicating that there were slow variations in frequency at different 


\section{A. Recording Sites}

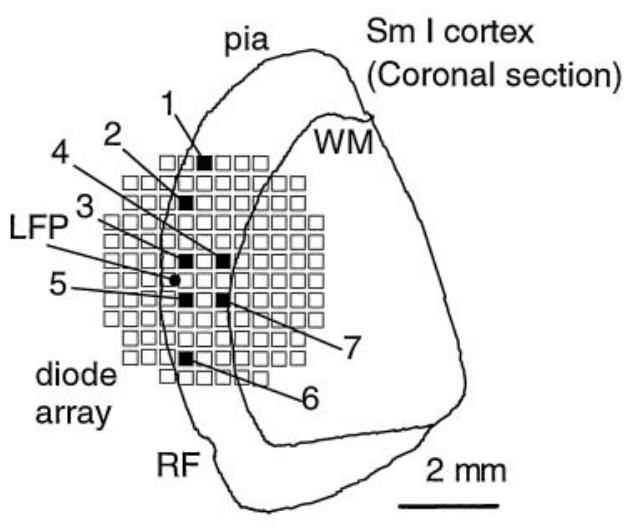

\section{Power Spectra}

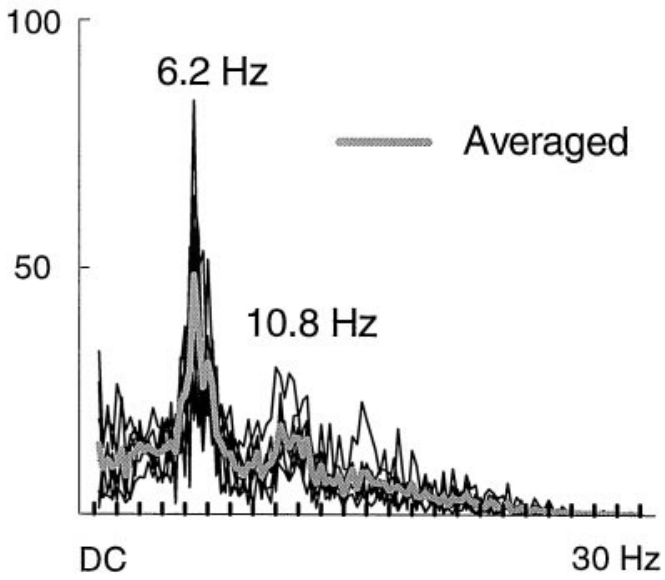

\section{B. Selected Recordings}

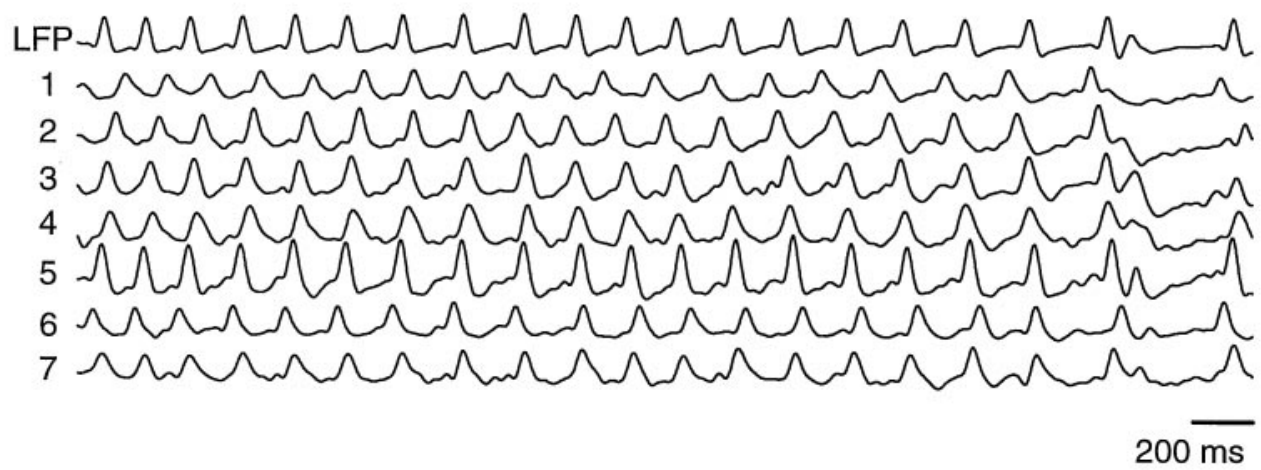

Figure 4. Multiple site recording of the oscillations. A, A section of cortical slice (Sm I area) imaged by $\sim 50$ neighboring photodetectors and one LFP electrode (in layer II). The imaging field was $\sim 4.5 \mathrm{~mm}$ in diameter. $R F$, Rhinal fissure; $W M$, white matter. $B$, Selected recording traces from the sites (solid squares in $A$ ). Both electrical and optical signals were low-pass filtered at $300 \mathrm{~Hz}$, and the amplitude of the traces has been normalized to emphasize the phase relationships. $C$, The power spectra of the data in $B$. The gray trace is the average of all individual FFTs, in which the major frequency peak was $6.2 \mathrm{~Hz}\left(\right.$ at $\left.26^{\circ} \mathrm{C}\right)$. A minor frequency peak $(\sim 10.8 \mathrm{~Hz})$ does not have a systematic representation in the raw data shown in $B$. This minor peak was also seen in in vivo LFP recordings (Nicolelis et al., 1995). This recording trial contained 16,000 frames of voltage-sensitive dye images. Subsequent figures (see Figs. 5-8) were made from selected sections of these same data.

locations (Fig. $5 B$ ). A plot of the time interval between sequential cycles at several locations (Fig. 6) indicated that for most cycles (cycles 1-6, 11-17) the frequency variations were highly correlated at different locations. However, in addition to a general tendency to slow down at all locations, in 72 out of 105 of the epochs we found sudden changes in the cycle-to-cycle period that occurred simultaneously at different locations (as illustrated in Fig. 6, cycles 7-10). The correlations between different locations during this sudden change in frequency followed fixed patterns. The change appeared to occur first at one side of the preparation and moved to the other side in approximately two cycles. Although it is difficult to understand what is happening from the plot in Figure 6, the pseudocolor images from the same data set (Fig. $7 C, I I$; see next section) offer a simple and elegant explanation for the sudden variation in frequency.

\section{Propagating activations during the oscillations}

The images derived from all the optical detectors revealed that the neurons activated during oscillations were not uniformly distributed over the slice. Instead, each oscillation peak appeared as a localized hot spot propagating across the cortex. In Figure 7 we made pseudocolor images based on the amplitude of the voltage-sensitive dye signals, from three time sections (I-III) within one epoch. When the LFP recordings in Figure $7 B$ are compared with the pseudocolor images in Figure $7 C$, it is obvious that each oscillation peak correlated to a propagating hot spot. In each oscillation cycle, when a hot spot passed by an LFP electrode (or an optical detector), a depolarization peak was detected. When many hot spots repeatedly passed a detector, a periodic depolarization was recorded that appeared as oscillations (Fig. $7 B$ ). Thus at each individual recording point, the activity could be defined as an oscillation by its periodicity in FFT and autocorrelations (Figs. $4 B, 5 B$ ). However, the images of the same data set revealed propagating hot spots (Fig. $7 C$ ), suggesting that the periodicity is in part determined by the propagation velocity.

The shape of the hot spots varied somewhat from cycle to cycle of the oscillations, and no particular pattern was correlated with each individual preparation. It is difficult to determine accurately the physical size of a hot spot from the amplitude pseudocolor image. However, using the $50 \%$ isopotential lines of the contour map, we determined that the size of the spots was $\sim 0.5 \mathrm{~mm}$ wide in a tangential direction and encompassed all cortical layers. The spots were relatively similar in size but somewhat irregular in 


\section{A. Cross-correlations}

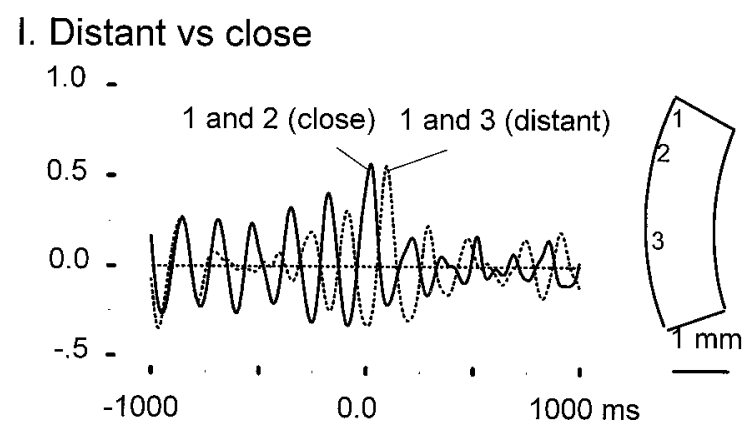

\section{Same column vs same layer}
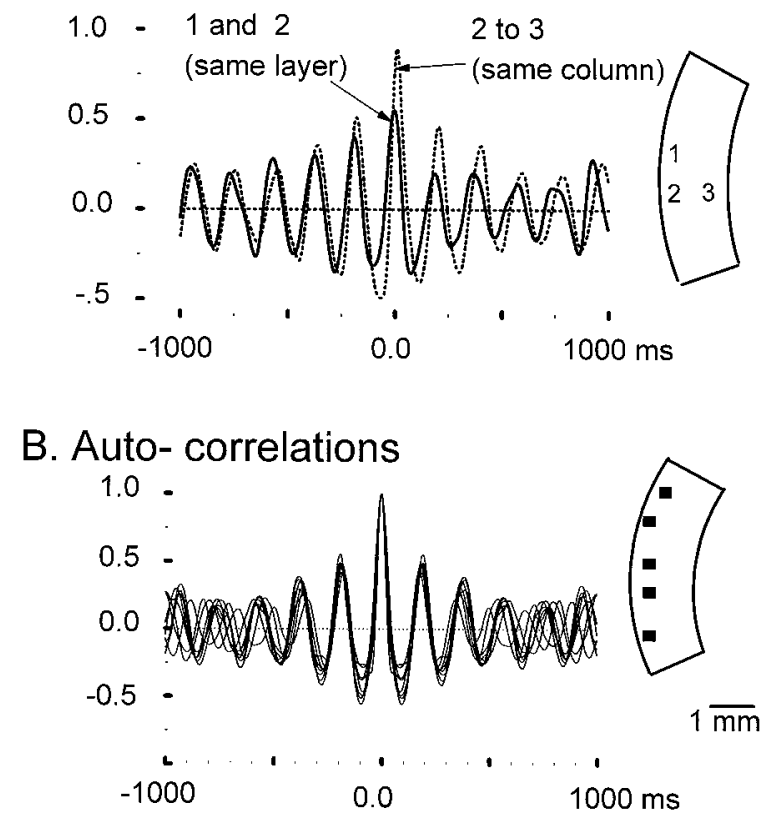

Figure 5. Auto- and cross-correlations. A, Cross-correlations. I, The amplitude of the correlations between the signals did not decrease significantly with distance. The solid and dotted traces are correlations between two detectors 800 and $3200 \mu \mathrm{m}$ apart, respectively. II, Signals from the same column (detectors 2, 3) have a somewhat larger correlation (dotted trace) than do the signals from the same layer but different columns (detectors 1, 2; solid trace). B, Autocorrelations of oscillation signals at five different locations (filled rectangles). At different locations, phase variations appear in outside peaks. The overall frequency was $6.2 \mathrm{~Hz}$.

shape (Fig. 7). The cycle-to-cycle variability of the shape and size suggested that these propagating hot spots were most likely dynamically organized. The hot spots were seen in the slices with different slicing angles from the barrel cortex. The isopotential contour did not appear to be correlated to the barrel patterns, suggesting that the activity is likely to be organized by distributed intracortical connections. Because the hot spots are likely to be dynamically organized and they are not a classical stationary oscillation, we give them the name "dynamic ensembles" to describe this propagating activation and to distinguish it from other kinds of population activity.

\section{Propagation, collisions, and jumps of dynamic ensembles}

For each cycle of an oscillation, one and occasionally two dynamic ensembles emerged and propagated away. The overall propaga-

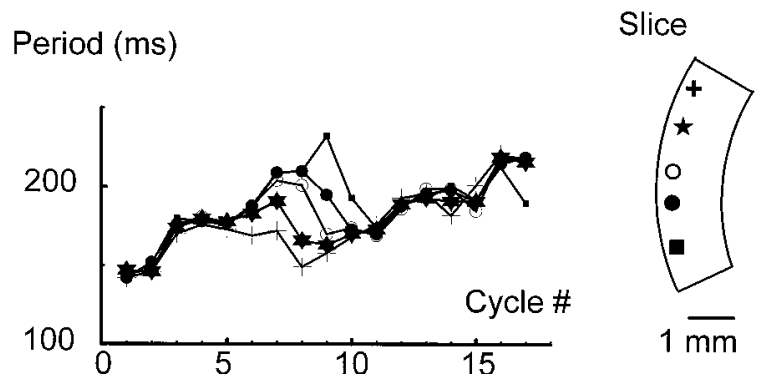

Figure 6. Variations in the cycle period at different locations. The cycle-to-cycle periodicity has sudden changes in some cycles (at cycles 7-10 in this epoch). At different locations the changes are in different directions. These kinds of sudden changes were very common. We examined 105 epochs from four preparations, and the sudden changes were found in 72 epochs.

tion velocity was $30 \pm 10 \mathrm{~mm} / \mathrm{sec}\left(\right.$ mean $\pm \mathrm{SD} ; n=15$; at $\left.26^{\circ} \mathrm{C}\right)$, and the distance traveled was usually larger than the field that was imaged (i.e., $>5 \mathrm{~mm}$ ). In a brain slice only two propagation directions are possible. A consecutive series of dynamic ensembles usually propagated in the same direction; $97 \%(n=200)$ kept the same direction as their predecessor. However, the propagating direction was not fixed. The direction could reverse in the middle of an oscillation epoch (Fig. 7B,C,I, III). When two dynamic ensembles emerged simultaneously at different locations, they sometimes propagated toward each other, resulting in collision and annihilation of the activity (Fig. $7 B, C, I I$ ). These collisions and changes in propagation direction explain why the oscillation frequencies varied drastically in the middle of some epochs (Fig. 6, cycles 7-10).

The propagation of excitation in cortical tissue often contains abrupt changes or "jumps" (Chervin et al., 1988; Wadman and Gutnick, 1993). We used the isopotential contour lines of the dynamic ensembles to examine variations in the propagation velocity. We found that the traveling course of each dynamic ensemble often had several jumps (Fig. $8 \mathrm{~B}$, arrows) that appeared as a rapid progression of the front edge of the isopotential contour line within a few milliseconds (Fig. 8A), followed by rapid withdrawal of the back edge (data not shown). Although the pattern of horizontal intracortical connections may be important in the propagation velocity of an excitation wave (Chervin et al., 1988; Wadman and Gutnick, 1993; Golomb and Amitai, 1997), in our data the locations of the jumps appeared to vary for each dynamic ensemble (Fig. $8 A, I-I I I$ ). It is thus more likely that the jumps in our image are dynamically distributed and not determined by the intracortical connectivity patterns.

\section{Neuronal activity within a dynamic ensemble}

The density of active neurons in a dynamic ensemble can be estimated by the amplitude of voltage-sensitive dye signals. A maximum response evoked by an electrical shock around the tip of a stimulating electrode was used as a standard of "maximum activation" of a neuron population. We assume that the majority of neurons near the electrode were simultaneously (within 1 msec) activated, orthodromically or antidromically, by the shock. The amplitude of the optical signal of a dynamic ensemble was measured from the same field of view and was $5-7 \%$ of the amplitude of maximum activation. This suggested a low-firing probability within a dynamic ensemble.

The low-firing probability in a dynamic ensemble is further confirmed by extracellular, multiunit recordings that directly 
A.

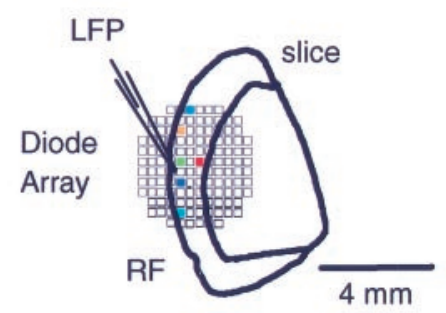

B.

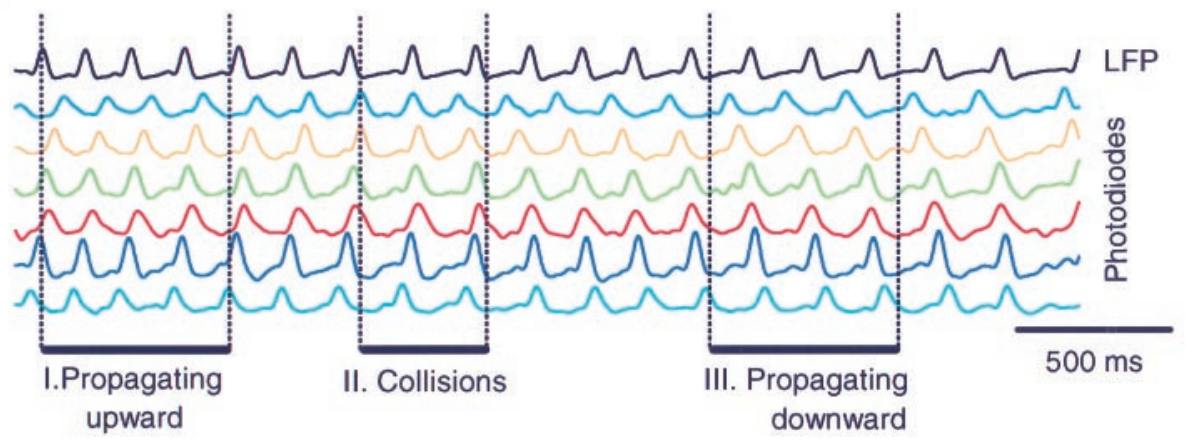

C.

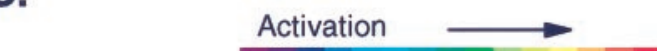

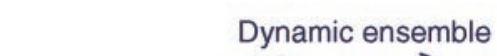

I. Propagating upward
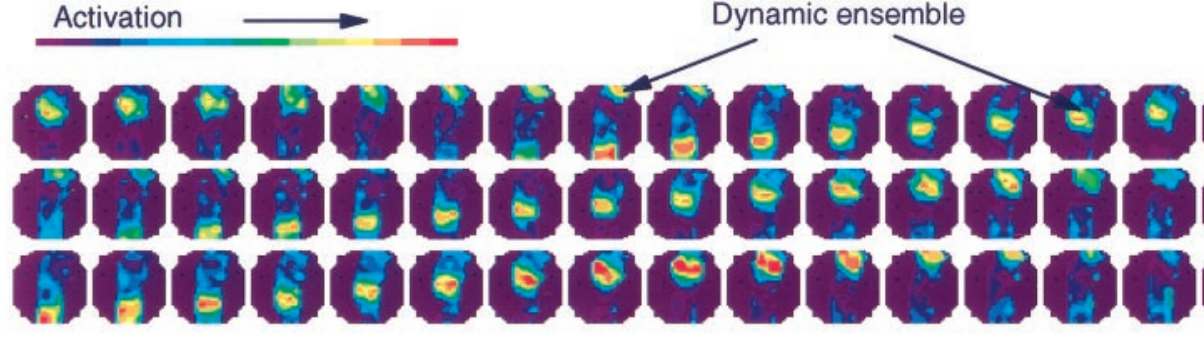

$10 \mathrm{~ms} /$ frame

$4 \mathrm{~mm}$

II. Collisions
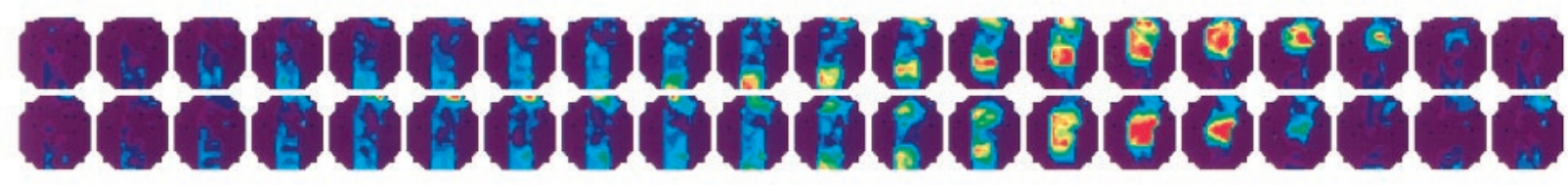

\section{Propagating}
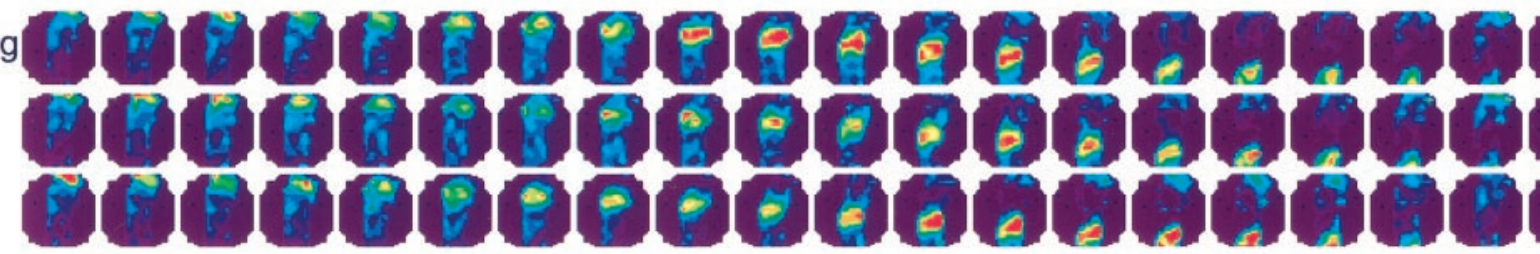

Figure 7. Propagating activation during the oscillations. $A$, The recording arrangement for the data in $B$ and $C . R F$, Rhinal fissure. $B$, Raw data from the LFP electrode and six optical detectors. Three sections from these data $(I, I I, I I I)$ are displayed in the pseudocolor images in $C$. $C$, Pseudocolor images from the three selected sections $I, I I$, and $I I I$ in $B$ displayed at $10 \mathrm{msec} /$ frame. In the images warm colors represent a larger depolarization. $I$, During each cycle of oscillation, one dynamic ensemble emerged and traveled across the field of view. Three dynamic ensembles appeared in this section and moved upward across the field of view. When they moved past the LFP electrode, a cycle of oscillation was detected on the electrode (black trace in $B$ ). II, Later $(0.5 \mathrm{sec})$, two dynamic ensembles appeared simultaneously (at the top and bottom of the field of view) and collided near the center. Two sequential collision events occurred during period $I I$. These collisions generated the dramatic change in oscillation frequency seen in Figure 6 . Activity was quickly annihilated after the collisions. III, After the collisions in period II, the propagation direction of the ensembles was reversed. The dynamic ensembles emerged from the top of the field of view and traveled downward. In all eight preparations imaged, the oscillations appeared as propagating activation-dynamic ensembles. The physical size of dynamic ensembles was estimated by the isopotential contour lines in the pseudocolor display and was $\sim 0.5 \mathrm{~mm}$ wide in the tangential direction.

measure the spiking density within a dynamic ensemble. One electrode can usually distinguish spikes from two to three neurons near its tip by the amplitude of the spikes. When a dynamic ensemble was passing by the region of the electrode, the neurons sampled by the electrode only fired one to three spikes [from two preparations, 50+ dynamic ensembles (J.-Y. Wu and L. Guan, unpublished observation)]. The same electrode could record many more spikes during the synchronous spike occurring at the beginning of an oscillation epoch. This showed that the electrode was able to detect the spikes from many neurons but only a few fired during each dynamic ensemble.

From limited multiunit recordings, we did not find spikes correlated in a millisecond time domain even though the two neurons were physically close to each other. This is consistent with the optical images because correlated firing of a large number of neurons in the millisecond domain would have generated a sharp spike in the optical signal.

\section{Distinguishing dynamic ensembles from epileptiform events}

We attempted to use voltage-sensitive dye imaging to distinguish dynamic ensembles from epileptiform events. The synchronous spike at the beginning of each oscillation epoch (Fig. 2B) had characteristics of epileptiform events in brain slices bathed in low [Mg] (Anderson et al., 1986; Zhang et al., 1996). This allowed us a direct comparison of the two different events in the same preparation. The synchronous spikes and dynamic ensembles appeared different in several ways. First, the synchronous spike appeared in the imaging as a spreading of an excitation wave, starting from a small initiation site and expanding to the entire 


\section{A. Front edge contour for three ensembles}
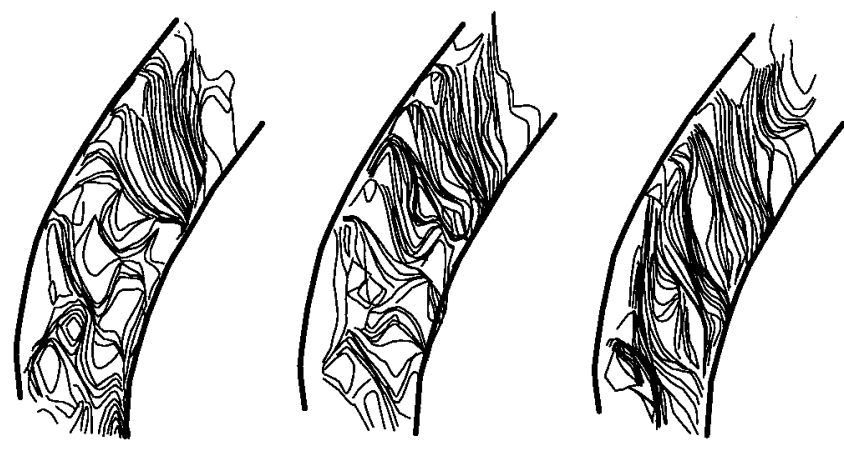

I

II

III

\section{B. Propagating velocity}

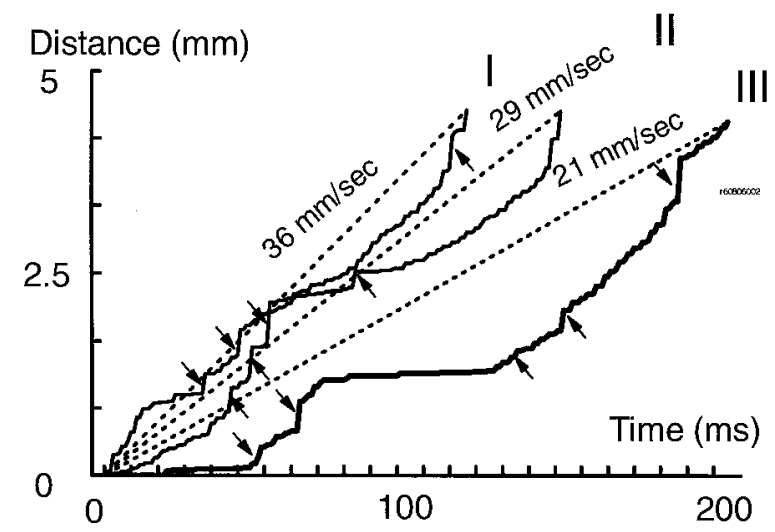

Figure 8. Details of the propagation of dynamic ensembles. $A$, The front edge contours of three dynamic ensembles are shown every 2 msec. The wide gaps between the contour lines suggest a sudden increase in propagating velocity. $I, I I$, Two consecutive dynamic ensembles jumped at similar (but not identical) locations. III, A dynamic ensemble that emerged $1 \mathrm{sec}$ later had quite different jump locations. $B$, The propagation velocity of the three dynamic ensembles (measured at cortical layer II) is shown. The overall velocities are 36,29 , and $21 \mathrm{~mm} / \mathrm{sec}$, composed of jumps (arrows) and slow propagation. We have examined the front edge contours from 10 dynamic ensembles from three preparations; the dynamically distributed jumps were seen in all 10 .

preparation (Fig. 9B, top four images). This pattern is similar to the imaging of epileptiform events described previously (Sutor et al., 1994; Albowitz and Kuhnt, 1995; Jackson and Scharfman, 1996; Tsau et al., 1998, 1999). In contrast, the activation of a dynamic ensemble was cohesive (not expanding), its physical size remaining relatively constant during propagation (Figs. $7 C, 9 B$, bottom row). Second, the amplitude of the dye signal of the synchronous spike was 5-20 times larger than that of dynamic ensembles (Fig. 9A), suggesting that the former had a higher density of active neurons. This is consistent with the extracellular electrode recordings, which showed that a synchronous spike had many more spikes than did a dynamic ensemble (data not shown). Third, epileptiform events generated a large peak in the intrinsic optical signal that was slow and wavelength independent (Fig. 9A, right) (see also Grinvald et al., 1986; Hochman et al., 1995; Yuste et al., 1997; Pazdalski et al., 1998). With dynamic ensembles this intrinsic signal was much smaller. Finally, epileptiform events and dynamic ensembles had different refractory periods. The former could not be evoked by an electrical stimulus applied at a rate higher than four times per minute, and the latter occurred at 5-8 $\mathrm{Hz}$ at $26^{\circ} \mathrm{C}$.

\section{Evoking dynamic ensembles in normal ACSF}

We attempted to show that a dynamic ensemble is an activity related to the intrinsic cortical circuits rather than a phenomenon only appearing during low $[\mathrm{Mg}]$ oscillations. We attempted to evoke dynamic ensembles when the cortical slice was bathed in normal ACSF, in which the neuronal network is not in a hyperexcitable condition. It is well known that in normal ACSF, the duration of a "passive" evoked response in the cortical slice is short [5-20 msec (Chagnac-Amitai and Connors, 1989)] and not self-sustaining. We propose, however, that with different ways of activating the cortical network, the excitation and inhibition balance may be altered, which would sustain the long-lasting activations of dynamic ensembles.

We used two kinds of electrical stimulation to evoke population activation in slices bathed in normal ACSF: (1) a conventional thin coaxial electrode, which evoked a high density of coactivation in a small area and which almost always elicited passive short-lasting evoked responses, and (2) a large tip single-pole electrode placed 100-300 $\mu \mathrm{m}$ above the tissue surface, which produced an electrical field over a large area, including all cortical layers (Fig. 10A). With this single-pole electrode, the area of activation and the density of activated neurons can be independently adjusted by changing the distance between the electrode and the tissue surface and by using different stimulus intensities. We can thus obtain a low level of activation (0.3-3 times the LFP response threshold) in a large area (1-2 $\mathrm{mm}$ in diameter). With this electrode and proper stimulus intensities, the evoked activity developed into long-lasting, propagating activations. In four preparations we delivered weak electrical stimuli at an interstimulus interval of $30 \mathrm{sec}$, and an LFP recording was used to detect the long-lasting population events (Fig. 10B, traces II, III). The optimum stimulus intensity was $\sim 0.5-0.7$ of the passive LFP response threshold. With an optimum stimulation intensity, $>90 \%$ of the stimuli evoked these long-lasting events. Increasing the stimulus intensity dramatically reduced the probability of inducing dynamic ensembles. When the intensity was increased by $20 \%$, only $\sim 40 \%$ of evoked activations became long-lasting; other responses were brief (Fig. 10B, trace I). Although the duration of the events varied from trial to trial, it was obvious that the activity was an all-or-none event. These data suggested that the longlasting events are self-sustained population activations, which can be evoked in a cortical network with unaltered excitability.

Optical imaging confirmed that these long-lasting events were similar to the dynamic ensembles in their size, shape, and active neuron density (Fig. 10C). Optical imaging also showed that the long-lasting activity always emerged in a location 1-2 mm away from the center of the stimulation point and in most of the cases 20-50 msec after the stimulus. This further suggested that the long-lasting events were dynamically organized by the cortical network and not a passive activation resulting from the electrical stimulus. On the basis of these observations, we extend our definition of dynamic ensembles to include both spontaneous and evoked population events sustained by the intrinsic cortical circuits that are dynamically organized and self-sustaining and have a low density of active neurons. 


\section{A. Difference in optical signals}

Figure 9. Dynamic ensembles are different from epileptiform events. A, Left, Middle, The epileptiform event was 10 times larger in dye signal amplitude (705 $\mathrm{nm}$ absorption) than were the dynamic ensembles (which are seen as an oscillation in one detector). Intrinsic optical signal also appears at $705 \mathrm{~nm}$, but with a much slower temporal course. We used high-pass filters (both analog and digital, with the RC time constant of $0.1 \mathrm{sec}$ ) to emphasize the voltagesensitive dye signals in these two panels. Right, The intrinsic optical signal of an epoch is shown. The signal is much larger and slower than are those on the left and in the middle. These were the same data shown on the left but displayed with a minimum high-pass filter and different time and gain scales. We examined three preparations in white light in which voltage-sensitive dye signals were undetectable. In these conditions the intrinsic optical signals were the same as that shown on the right. B, Top row, An epileptiform event was evoked by an electrical stimulus (Stim) at the center of the field of view (see diagram on the right). This event expanded to the entire field of view in $\sim 30$ msec. Bottom Row, A dynamic ensemble emerged from the same cortical area (same field of view, $7 \mathrm{sec}$ later) and remained cohesive for at least $80 \mathrm{msec}$. The two rows are displayed with different pseudocolor scales to show the propagation of both events.

\section{DISCUSSION}

In this report we have characterized the population activation during $7-10 \mathrm{~Hz}$ oscillations. We also found that similar activity could be elicited in conditions of normal excitability (slice in normal ACSF). We name this dynamically organized, selfsustaining, and propagating activation dynamic ensembles.

\section{Dynamic ensembles and population oscillations}

We have demonstrated that the same population events can be described either as oscillations at an individual location (measured optically or with electrodes) or as propagating dynamic ensembles (measured by imaging) (Figs. 7, 10B). The ubiquitous existence of oscillations (Tank et al., 1994; Singer and Gray, 1995) and the link between population oscillations and dynamic ensembles raise the possibility that dynamic ensembles might be a common form of population event in the neocortical neuronal network.

Slice preparations from different parts of the brain often have an oscillation frequency that is similar to those found in vivo. These oscillations include $6-10 \mathrm{~Hz}$ spindles in the thalamus (Bal et al., 1995; Kim et al., 1995), 7-10 Hz in somatosensory cortex (Silva et al., 1991) (this paper), 20-80 Hz in auditory cortex (Metherate and Cruikshank, 1999), 10-50 Hz in other parts of the neocortex (Llinas et al., 1991), and $40 \mathrm{~Hz}$ in the hippocampus (Whittington et al., 1995). In a few slice preparations, the spatiotemporal patterns of oscillations have been examined. Field potential measurements from ferret thalamus slices indicated that the $5-10 \mathrm{~Hz}$ spindle oscillations also appear as propagating waves

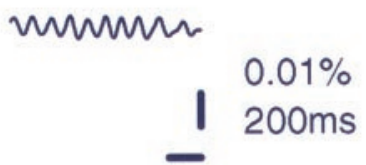

dynamic ensembles, dye signal

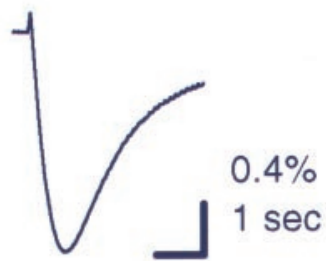

Epileptiform,

intrinsic signal

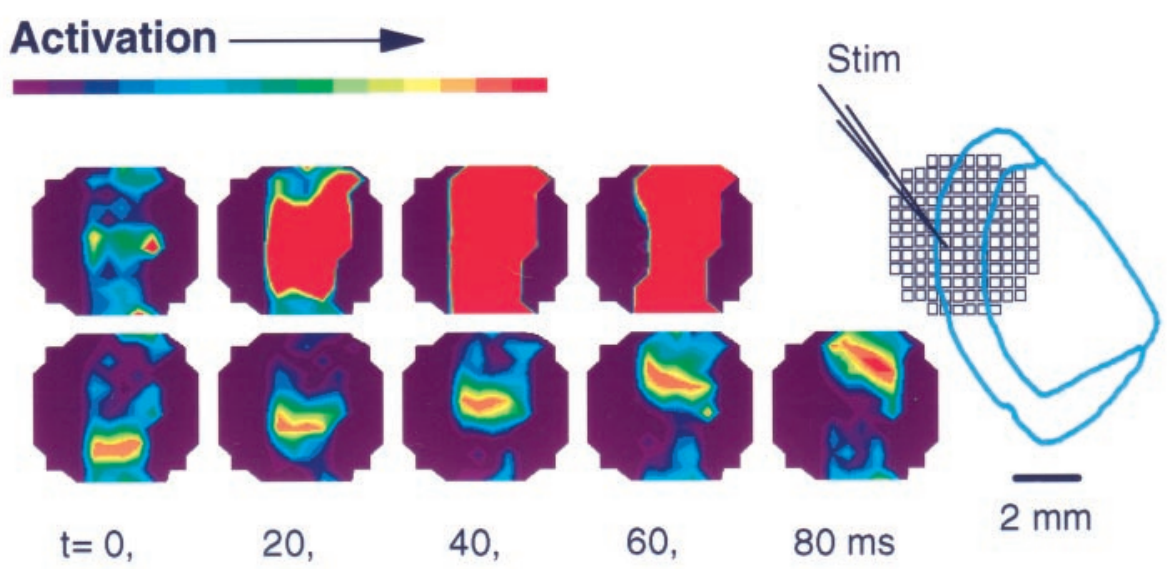

(Kim et al., 1995). These thalamic spindle waves had a similar frequency but propagated 10 times slower than did the dynamic ensembles we have seen in the cortex. Because propagation velocity determines the wavelength or distance between dynamic ensembles, it would be interesting to compare the images of oscillation waves generated by different mechanisms, e.g., "lurching" thalamic spindle versus "continuous" cortical waves (Golomb et al., 1996; Rinzel et al., 1998). This would allow us to examine whether dynamic ensembles are of more general occurrence in different local circuits.

Imaging of in vivo oscillations has shown that they also appeared as propagating excitation waves (Delaney et al., 1994; Kleinfeld et al., 1994; Lam, 1997; Prechtl et al., 1997). The rostral oscillation in turtle olfactory bulb has a simple propagating pattern (Y.-W. Lam, L. B. Cohen, M. Wachowiak, and M. R. Zochowski, unpublished observations), which is strikingly similar to our in vitro observations. At each cycle of the rostral oscillation, a "blob" of activation emerged from one location and propagated with a certain velocity and direction in the bulb. The physical size, active neuron density, and propagation velocity of the blob were very similar to those of the dynamic ensembles that we observed in cortical slices. Visually induced oscillations in turtle cortex have multiple frequency domains, with propagating blobs representing each frequency domain (Prechtl et al., 1997). Oscillations in the somatosensory cortex of behaving rats (7-10 $\mathrm{Hz}$ ) are also organized in epochs during whisking, and the oscillations also appear as traveling waves (Nicolelis et al., 1995). Unfortunately, descriptions of the spatial pattern of in vivo oscil- 


\section{A. Stimulation at low intensity in a large}

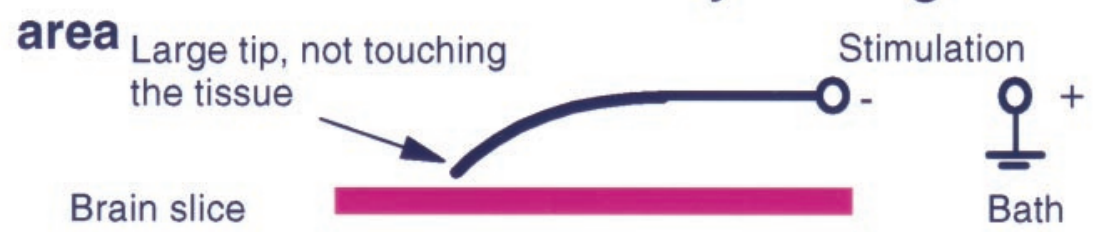

\section{B. Passive(I) and Long-lasting(II and III) responses (in LFP)}
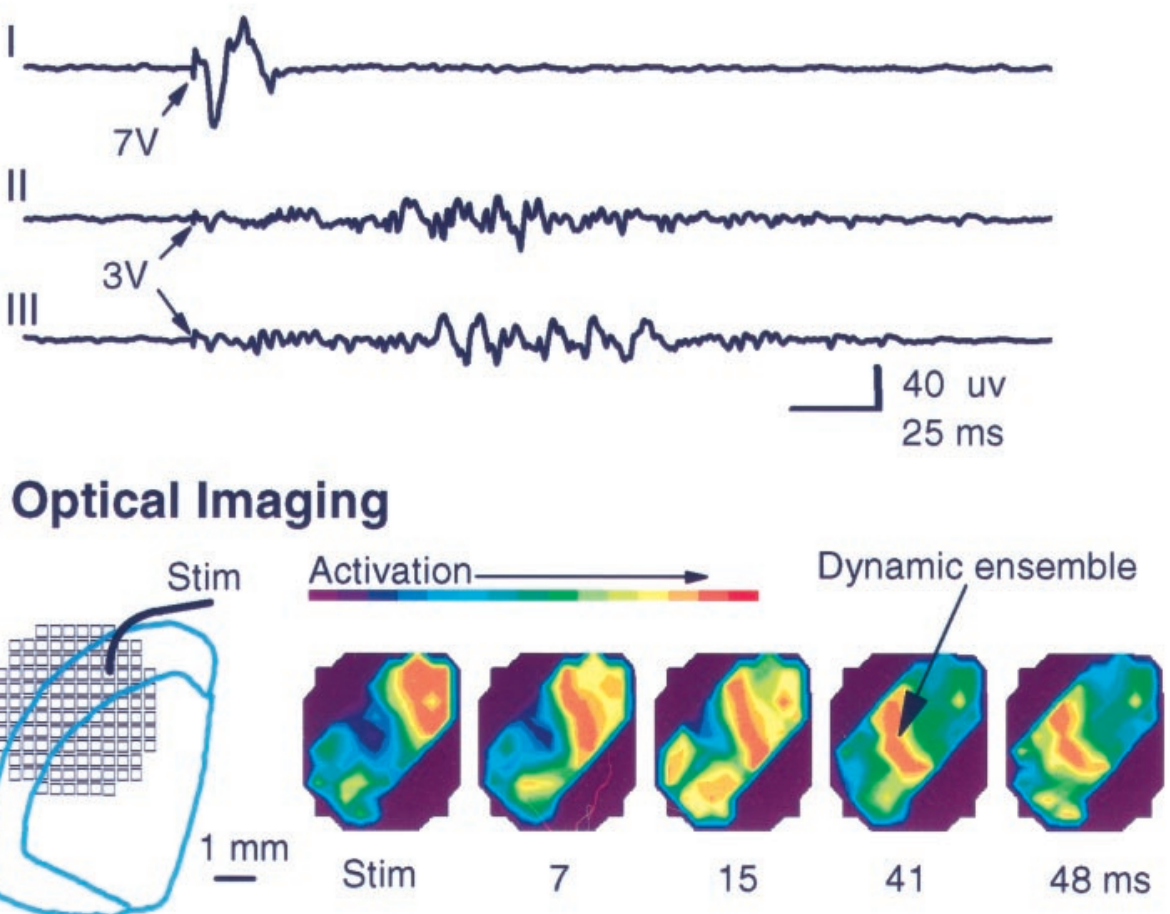
msec and propagated downward in the image field. The slice was perfused in normal ACSF, and the optical signal had a small amplitude compared with that of an epileptiform spike. Note the propagation velocity was $\sim 6-10 \mathrm{~mm} / \mathrm{sec}(n=10$, measured from 2 preparations), significantly slower than the

lations have been limited because no imaging data from mammalian cortex have been available.

Going from an intact cortex to a cortical slice is a significant reduction from a large three-dimensional cortical network to a relatively small and virtually two-dimensional network. This reduces the number of possible initiators of oscillations and the connections between them. Thus a propagating wave would encounter fewer interactions with activation initiated by other initiators. The propagation of spindle waves in thalamic slices (Kim et al., 1995) might have much fewer collisions than in the intact thalamus (Contreras et al., 1997). Thus our images of dynamic ensembles might only present a simplified situation of oscillatory events in vivo.

Population oscillations have been proposed to serve as a timing signal (von der Malsburg, 1985; Singer, 1993), as referencecarrying waves for temporal coding (Hopfield, 1995) or for arousal or attention (Steriade et al., 1991; MacKay and Mendonca, 1995; Munk et al., 1996). Our data showed that during oscillations different locations were activated sequentially as dynamic ensembles propagated. This presents questions about the

Figure 10. Dynamic ensembles evoked in normal ACSF. $A$, Schematic diagram of a single-pole electrode placed above the slice is shown. This stimulation method can evoke a low density of activated neurons distributed in a large area including several cortical layers. The intensity and the size of the area can be independently controlled by adjusting stimulation voltage and the distance between the electrode and the slice. $B$, Long-lasting response in local field potential evoked by weak electrical shocks is shown. In this preparation an electrode was placed on top of layer IV, $\sim 80 \mu \mathrm{m}$ above the preparation. The interstimulus interval was $30 \mathrm{sec}$. Trace I, With stimulus intensity of $7 \mathrm{~V}$ (duration = $0.05 \mathrm{msec}$, for all stimuli), just above the threshold of the passive LFP response, a passive response (short and more synchronized) was evoked. Traces II, III, With intensity reduced to $3 \mathrm{~V}$, long-lasting activity was evoked. The long-lasting responses were allor-none events. In this preparation, $98 \%(n=$ 120) of $3 \mathrm{~V}$ stimuli evoked a long-lasting response. When the stimulus was increased to $5.5 \mathrm{~V}, \sim 60 \%$ of the stimuli failed to evoke a long-lasting response. At $7 \mathrm{~V}$, all the stimuli $(n=20)$ evoked the passive response (trace $I$ ). Traces II and III are consecutive trials, illustrating the variation of the long-lasting response. $C$, The long-lasting response was spatially confined (not expanding) and propagating, similar to dynamic ensembles (Fig. 7). A weak electrical shock $(\sim 10 \mathrm{~V} \times 0.05$ msec) from an electrode placed $100 \mu \mathrm{m}$ above the preparation [at the upper right corner of the image field (see diagram on the left)] induced activity over a large area including all layers (Stim image on the left). Seven milliseconds later, a long-lasting, propagating activation emerged next to the area activated by the stimulus (second image from the left). This activity had an amplitude that was similar to that of the dynamic ensembles observed during $7-10 \mathrm{~Hz}$ oscillations (e.g., Fig.

7). The activity remained cohesive for $\sim 40$

\section{Optical Imaging}
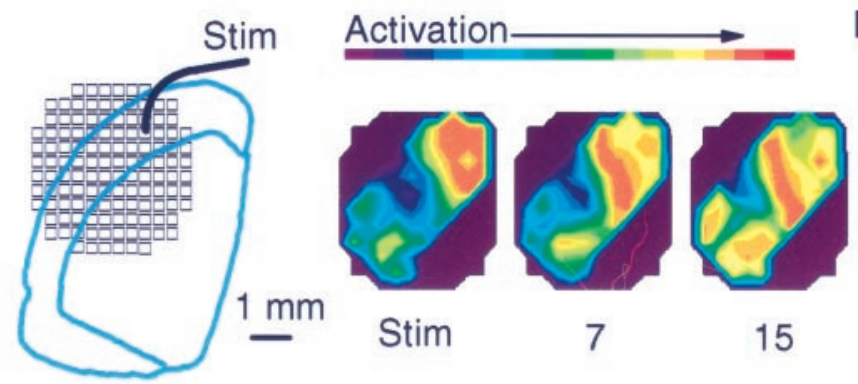
propagation during oscillations. Stim, Stimulus.

$25 \mathrm{~ms}$ definition of synchrony in the hundred millisecond time domain and how such synchrony is used for cortical processing.

\section{Dynamic ensembles and cortical neuronal organization}

The interaction between cortical cellular organization (e.g., columns) and propagating dynamic ensembles remains to be explored. Our data suggested that the correlation within a cortical column is better than that between neighboring columns (Fig. 5). It might be speculated that a dynamic ensemble consists of activity in one or several cortical columns and that this activity jumps from one column to another. However, we noticed that the propagation patterns of the dynamic ensembles (Fig. 8) were not consistent with the speculation that dynamic ensembles jump from barrel to barrel. Also, in tangential slices from the somatosensory areas, we could not find a correlation between the activity of dynamic ensembles and the whisker barrels ( $\mathrm{Wu}$ and Guan, unpublished observation). [Chervin et al. (1988) also found no correlation between barrel patterns and propagation jumps.] These observations suggested that dynamic ensembles 
were more likely to be organized by intrinsic (local) circuits rather than by afferent-related cellular organizations.

The organization of intrinsic circuits in the neocortex is relatively similar in different areas (Shepherd, 1988; Douglas and Martin, 1990). This similarity [the "canonical" circuit (Douglas and Martin, 1990)] suggests universal rules for organizing local population activation in different cortices. As a continuation of this work, it would be interesting to compare oscillations and evoked population events in different cortical areas, to test whether dynamic ensembles can be evoked in all cortical tissues.

\section{The nature of activation in a dynamic ensemble}

We estimate that a dynamic ensemble has only $\sim 5-7 \%$ of the maximum activation of the cortical network. The voltagesensitive dye signals come from a combination of spikes and synaptic potentials. If we assume that our signals were a linear summation of spikes and synaptic potentials in the population (Wu and Cohen, 1993), then one spike $(100 \mathrm{mV})$ of 1 neuron would contribute equally with 10 neurons that have a $10 \mathrm{mV}$ depolarization. At one extreme, we could assume that all signals were contributed by spikes, so that $5-7 \%$ of the total population was firing simultaneously in each sampling period. Thus during the $200 \mathrm{msec}$ duration of a dynamic ensemble, each activated neuron would fire $\sim 10$ times. However, extracellular unit recordings revealed that each neuron only fired one or two spikes. Therefore it seems likely that subthreshold depolarizations of many cells are an important contributor to the dynamic ensemble signals. We thus speculate that a dynamic ensemble is organized as a domain of moderately depolarized neurons in which some neurons will increase their firing probability to reach one to two spikes per event. This kind of organization is similar to the spontaneous population coactivation observed in developing cortex (Yuste et al., 1992) and retina (Wong et al., 1995; Feller et al., 1997).

\section{Self-sustainability: functional significance}

The activation of dynamic ensembles lasted much longer than an ordinary evoked response. We considered this activation to be self-sustaining, i.e., sustaining a certain level of activity in the cortex without afferent input. We hypothesize that selfsustainability is a population property of a reciprocally activating neuronal network. The development of a dynamic ensemble might follow certain population rules, e.g., depend on the size of the activation, the density of active neurons in the area, and the temporal pattern of the input (cf. Golomb and Amitai, 1997). In this report we evoked long-lasting activity by activating a low density of neurons in a large area involving all cortical layers (Fig. 10). The all-or-none nature of a population response supports our speculation that the activity is self-sustained. This kind of longlasting evoked response can also be evoked by stimulating thalamocortical afferents (Metherate and Cruikshank, 1999).

How dynamic ensembles interact with evoked events remains to be explored. In intact brain, dynamic ensembles may be evoked by sensory input or emerge during evoked oscillations. This would allow spatial interactions between different ensembles and temporal interactions between concurrent input and dynamic ensembles evoked by previous input. Self-sustained activity in dynamic ensembles may provide an uneven local excitability, in which the same input may elicit a higher (or lower) population firing rate within an ensemble than in the regions outside of it. This suggests a mechanism for cortical reverberation (Hebb, 1949; Amit, 1995), a process in which a local network can amplify a weak input with a compatible spatiotemporal pattern and reject a strong input with incompatible patterns.
The existence of dynamically organized population modules has been postulated as a "cell assembly" (Hebb, 1949; Amit, 1995; Nicolelis et al., 1997) that reverberates with afferent input and may play a role in sensory discrimination (Zhang, 1996), temporary information storage, attentional modulation of sensory processing, and transition from short- to long-term plasticity (Rauschecker, 1995). Our study of dynamic ensembles suggests that cortical intrinsic circuits can serve as a host for self-sustained and dynamically organized population activity. Such a form of activity could be used to "bind" afferent input distributed in time and space.

\section{REFERENCES}

Albowitz B, Kuhnt U (1991) Spatio-temporal distribution of epileptiform potentials in the hippocampal slice: recordings with voltagesensitive dyes. Eur J Neurosci 3:570-586.

Albowitz B, Kuhnt U (1995) Epileptiform activity in the guinea-pig neocortical slice spreads preferentially along supragranular layersrecordings with voltage-sensitive dyes. Eur J Neurosci 7:1273-1284.

Amit DJ (1995) The Hebbian paradigm reintegrated: local reverberations as internal representations. Behav Brain Sci 18:617-657.

Anderson WW, Lewis DV, Swartzwelder HS, Wilson WA (1986) Magnesium-free medium activates seizure-like events in the rat hippocampal slice. Brain Res 398:215-219.

Arieli A, Sterkin A, Grinvald A, Aertsen A (1996) Dynamics of ongoing activity: explanation of the large variability in evoked cortical responses. Science 273:1868-1871.

Bal T, von Krosigk M, McCormick DA (1995) Role of the ferret perigeniculate nucleus in the generation of synchronized oscillations in vitro. J Physiol (Lond) 483:665-685.

Chagnac-Amitai Y, Connors BW (1989) Horizontal spread of synchronized activity in neocortex and its control by GABA-mediated inhibition. J Neurophysiol 61:747-758.

Chervin RD, Pierce PA, Connors BW (1988) Periodicity and directionality in the propagation of epileptiform discharges across neocortex. J Neurophysiol 60:1695-1713.

Churchland PS, Sejnowski TJ (1992) The computational brain. Cambridge, MA: MIT.

Colom LV, Saggau P (1994) Spontaneous interictal-like activity originates in multiple areas of the CA2-CA3 region of hippocampal slices. J Neurophysiol 71:1574-1585.

Connors BW, Gutnick MJ (1990) Intrinsic firing patterns of diverse neocortical neurons. Trends Neurosci 13:99-104.

Contreras D, Destexhe A, Sejnowski TJ, Steriade M (1997) Spatiotemporal patterns of spindle oscillations in cortex and thalamus. J Neurosci 17:1179-1196.

Crick F (1996) Visual perception: rivalry and consciousness [news; comment]. Nature 379:485-486.

Delaney KR, Gelperin A, Fee MS, Flores JA, Gervais R, Tank DW, Kleinfeld D (1994) Waves and stimulus-modulated dynamics in an oscillating olfactory network. Proc Natl Acad Sci USA 91:669-673.

Demir R, Haberly LB, Jackson MB (1998) Voltage imaging of epileptiform activity in slices from rat piriform cortex: onset and propagation. J Neurophysiol 80:2727-2742.

Douglas RJ, Martin KAC (1990) Neocortex. In: The synaptic organization of the brain (Shepherd GM, ed), pp 401-435. New York: Oxford UP.

Engel AK, Konig P, Kreiter AK, Singer W (1991) Interhemispheric synchronization of oscillatory neuronal responses in cat visual cortex. Science 252:1177-1179.

Feller MB, Butts DA, Aaron HL, Rokhsar DS, Shatz CJ (1997) Dynamic processes shape spatiotemporal properties of retinal waves. Neuron 19:293-306.

Flint AC, Connors BW (1996) Two types of network oscillations in neocortex mediated by distinct glutamate receptor subtypes and neuronal populations. J Neurophysiol 75:951-957.

Freeman WJ (1995) The Hebbian paradigm reintegrated: local reverberations as internal representations. Behav Brain Sci 18:631.

Gerstein GL, Aertsen AM (1985) Representation of cooperative firing activity among simultaneously recorded neurons. J Neurophysiol 54:1513-1528.

Gerstein GL, Bedenbaugh P, Aertsen MH (1989) Neuronal assemblies. IEEE Trans Biomed Eng 36:4-14. 
Glaser D, Hildesheim R, Shoham D, Grinvald A (1998) Optical imaging with new voltage-sensitive dyes reveals that sudden illumination changes delay the onset of oriental tuning in cat visual cortex. Soc Neurosci Abstr 24:10.

Golomb D, Amitai Y (1997) Propagating neuronal discharges in neocortical slices: computational and experimental study. J Neurophysiol 78:1199-1211.

Golomb D, Wang XJ, Rinzel J (1996) Propagation of spindle waves in a thalamic slice model. J Neurophysiol 75:750-769.

Gray CM (1994) Synchronous oscillations in neuronal systems: mechanisms and functions. J Comput Neurosci 1:11-38.

Grinvald A, Manker A, Segal M (1982) Visualization of the spread of electrical activity in rat hippocampal slices by voltage-sensitive optical probes. J Physiol (Lond) 333:269-291.

Grinvald A, Lieke E, Frostig RD, Gilbert CD, Wiesel TN (1986) Functional architecture of cortex revealed by optical imaging of intrinsic signals. Nature 324:361-364.

Hebb DO (1949) The organisation of behaviour. Philadelphia: Wiley.

Hochman DW, Baraban SC, Owens JW, Schwartzkroin PA (1995) Dissociation of synchronization and excitability in furosemide blockade of epileptiform activity. Science 270:99-102.

Hopfield JJ (1995) Pattern recognition computation using action potential timing for stimulus representation. Nature 376:33-36.

Jackson MB, Scharfman HE (1996) Positive feedback from hilar mossy cells to granule cells in the dentate gyrus revealed by voltage-sensitive dye and microelectrode recording. J Neurophysiol 76:601-616.

Kim U, Bal T, McCormick DA (1995) Spindle waves are propagating synchronized oscillations in the ferret LGNd in vitro. J Neurophysiol 74:1301-1323.

Kleinfeld D, Delaney KR (1996) Distributed representation of vibrissa movement in the upper layers of somatosensory cortex revealed with voltage-sensitive dyes. J Comp Neurol 375:89-108.

Kleinfeld D, Delaney KR, Fee MS, Flores JA, Tank DW, Gelperin A (1994) Dynamics of propagating waves in the olfactory network of a terrestrial mollusk: an electrical and optical study. J Neurophysiol 72:1402-1419.

Lam YW (1997) Voltage sensitive dye recording of odor elicited oscillations in the turtle olfactory bulb. Soc Neurosci Abstr 23:500.

Llinas R, Ribary U (1993) Coherent 40-Hz oscillation characterizes dream state in humans. Proc Natl Acad Sci USA 90:2078-2081.

Llinas RR, Grace AA, Yarom Y (1991) In vitro neurons in mammalian cortical layer 4 exhibit intrinsic oscillatory activity in the $10-$ to $50-\mathrm{Hz}$ frequency range. Proc Natl Acad Sci USA [Erratum (1991) 88:3510] 88:897-901.

London JA, Cohen LB, Wu JY (1989) Optical recordings of the cortical response to whisker stimulation before and after the addition of an epileptogenic agent. J Neurosci 9:2182-2190.

MacKay WA, Mendonca AJ (1995) Field potential oscillatory bursts in parietal cortex before and during reach. Brain Res 704:167-174.

Malonek D, Grinvald A (1996) Interactions between electrical activity and cortical microcirculation revealed by imaging spectroscopy: implications for functional brain mapping. Science 272:551-554.

Metherate R, Cruikshank SJ (1999) Thalamocortical inputs trigger a propagating envelope of gamma-band activity in auditory cortex, in vitro. Exp Brain Res 126:160-174.

Munk MH, Roelfsema PR, Konig P, Engel AK, Singer W (1996) Role of reticular activation in the modulation of intracortical synchronization. Science 272:271-274.

Murthy VN, Fetz EE (1996) Oscillatory activity in sensorimotor cortex of awake monkeys: synchronization of local field potentials and relation to behavior. J Neurophysiol 76:3949-3967.

Nelson DA, Katz LC (1995) Emergence of functional circuits in ferret visual cortex visualized by optical imaging. Neuron 15:23-34.

Nicolelis MAL, Baccala LA, Lin RCS, Chapin JK (1995) Sensorimotor encoding by synchronous neural ensemble activity at multiple levels of the somatosensory system. Science 268:1353-1358.

Nicolelis MAL, Fanselow EE, Ghazanfar AA (1997) Hebb's dream: the resurgence of cell assemblies. Neuron 19:219-221.

Palm G (1990) Cell assemblies as a guideline for brain research. Concepts Neurosci 1:133-137.

Paxinos G, Watson C (1986) The rat brain in stereotaxic coordinates, Ed 2. San Diego: Academic.

Pazdalski PS, Tsau Y, Wu J-Y (1998) Light scattering in cortical tissue as an indicator of normal and epileptiform activity: an in vitro study. Soc Neurosci Abstr 24:1059.
Peterson BE, Goldreich D, Merzenich MM (1998) Optical imaging and electrophysiology of rat barrel cortex. I. Responses to small singlevibrissa deflections. Cereb Cortex 8:173-183.

Prechtl JC, Cohen LB, Pesaran B, Mitra PP, Kleinfeld D (1997) Visual stimuli induce waves of electrical activity in turtle cortex. Proc Natl Acad Sci USA 94:7621-7626.

Rauschecker JP (1995) Reverberations of Hebbian thinking. Behav Brain Sci 18:642-643.

Rinzel J, Terman D, Wang X, Ermentrout B (1998) Propagating activity patterns in large-scale inhibitory neuronal networks. Science 279: $1351-1355$

Shepherd GM (1988) A basic circuit for cortical organization. In: Perspectives on memory research (Gazzaniga MC, ed), pp 93-134. Cambridge, MA: MIT.

Silva LR, Amitai Y, Connors BW (1991) Intrinsic oscillations of neocortex generated by layer 5 pyramidal neurons. Science 251:432-435.

Singer W (1985) Activity-dependent self-organization of the mammalian visual cortex. In: Models of the visual cortex (Rose D, Dobson VG, eds), pp 123-136. Chichester, United Kingdom: Wiley.

Singer W (1993) Neuronal representations, assemblies and temporal coherence. Prog Brain Res 95:461-474.

Singer W, Gray CM (1995) Visual feature integration and the temporal correlation hypothesis. Annu Rev Neurosci 18:555-586.

Steriade M, Dossi RC, Nunez A (1991) Network modulation of a slow intrinsic oscillation of cat thalamocortical neurons implicated in sleep delta waves: cortically induced synchronization and brainstem cholinergic suppression. J Neurosci 11:3200-3217.

Steriade M, McCormick DA, Sejnowski TJ (1993) Thalamocortical oscillations in the sleeping and aroused brain. Science 262:679-685.

Sutor B, Hablitz JJ, Rucker F, ten Bruggencate G (1994) Spread of epileptiform activity in the immature rat neocortex studied with voltage-sensitive dyes and laser scanning microscopy. J Neurophysiol 72:1756-1768.

Tank DW, Gelperin A, Kleinfeld D (1994) Odors, oscillations, and waves: does it all compute? Science 265:1819-1820.

Tsau Y, Guan L, Wu J-Y (1998) Initiation of spontaneous epileptiform activity in the neocortical slice. J Neurophysiol 80:978-982.

Tsau Y, Guan L, Wu J-Y (1999) Epileptiform activity can be initiated in various neocortical layers: an optical imaging study. J Neurophysiol, in press.

Ts'o DY, Frostig RD, Lieke EE, Grinvald A (1990) Functional organization of primate visual cortex revealed by high resolution optical imaging. Science 249:417-420.

von der Malsburg C (1985) Nervous structures with dynamical links. Ber Bunsenges Phys Chem 89:703-710.

Wadman WJ, Gutnick MJ (1993) Non-uniform propagation of epileptiform discharge in brain slices of rat neocortex. Neuroscience $52: 255-262$

Wang G, Tanaka K, Tanifuji M (1996) Optical imaging of functional organization in the monkey inferotemporal cortex. Science 272:1665-1668.

Whittington MA, Traub RD, Jefferys JG (1995) Synchronized oscillations in interneuron networks driven by metabotropic glutamate receptor activation. Nature 373:612-615.

Wong RO, Chernjavsky A, Smith SJ, Shatz CJ (1995) Early functional neural networks in the developing retina. Nature 374:716-718.

Wu J-Y, Cohen LB (1993) Fast multisite optical measurement of membrane potential. In: Biological techniques: fluorescent and luminescent probes for biological activity (Mason WT, ed), pp 389-404. New York: Academic.

Wu J-Y, Cohen LB, Falk CX (1999) Fast multisite optical measurement of membrane potential: with two examples. In: Fluorescent and luminescent probes for biological activity (Mason WT, ed). New York: Academic, in press.

Yuste R, Peinado A, Katz LC (1992) Neuronal domains in developing neocortex. Science 257:665-669.

Yuste R, Tank DW, Kleinfeld D (1997) Functional study of the rat cortical microcircuitry with voltage-sensitive dye imaging of neocortical slices. Cereb Cortex 7:546-558.

Zhang K (1996) Representation of spatial orientation by the intrinsic dynamics of the head-direction cell ensemble: a theory. J Neurosci 16:2112-2126.

Zhang YF, Gibbs 3rd JW, Coulter DA (1996) Anticonvulsant drug effects on spontaneous thalamocortical rhythms in vitro: ethosuximide, trimethadione, and dimethadione. Epilepsy Res 23:15-36. 